\title{
Impaired left-ventricular cardiac function in male GPR30-deficient mice
}

\author{
MARTINA DELBECK ${ }^{1}$, STEFAN GOLZ ${ }^{1}$, RICHARDUS VONK ${ }^{2}$, WIEBKE JANSSEN ${ }^{5}$, \\ TIM HUCHO $^{4}$, JÖRG ISENSEE ${ }^{4}$, STEFAN SCHÄFER ${ }^{6}$ and CHRISTIANE OTTO ${ }^{3}$ \\ ${ }^{1}$ Cardiology Research, Bayer Schering Pharma AG, 42096 Wuppertal; ${ }^{2}$ Global Drug Discovery Statistics, \\ and ${ }^{3}$ Women's Healthcare Research, Bayer Schering Pharma AG, 13342 Berlin; ${ }^{4}$ Max Planck \\ Institute for Molecular Genetics, 14195 Berlin; ${ }^{5}$ Max Planck Institute for Heart and Lung Research, \\ 61231 Bad Nauheim, Germany; ${ }^{6}$ Synthon B.V., 6503 GN Nijmegen, Netherlands
}

Received August 27, 2010; Accepted October 26, 2010

DOI: $10.3892 / \mathrm{mmr} .2010 .402$

\begin{abstract}
G-protein-coupled receptor 30 (GPR30) has been reported to act as a membrane-bound estrogen receptor that is involved in the mediation of non-genomic estradiol signalling. In this study, we demonstrated that male, but not female, GPR30-deficient mice suffer from impaired left-ventricular cardiac function. Left ventricles from male mutant mice were enlarged. There were no malformations in the valves or outflow tract of the heart. Both the contractility and relaxation capacity of the left ventricle were reduced, leading to increased left-ventricular end-diastolic pressure in GPR30-deficient mice. In conclusion, our data support a role for GPR30 in the gender-specific aspects of heart failure.
\end{abstract}

\section{Introduction}

In heart failure (HF), there are many gender-specific differences regarding the pathophysiology, clinical presentation, course and responsiveness to therapy of the disease (1). The incidence of HF is higher in men than in women at all ages (2). While women suffering from heart failure tend to develop concentric hypertrophy with almost preserved systolic function, male patients present dilated ventricles and reduced systolic cardiac function (1). Different hormonal milieus and risk factors in women and men might explain, to some extent, the observed gender-specific differences in $\operatorname{HF}(1,2)$. Nevertheless, further identification of the genes involved in the gender-specific aspects of heart failure is required to facilitate the development of novel patient-tailored therapies.

In this study, we identified GPR30 as a novel candidate gene for the gender-specific aspects of HF. GPR30 is a

Correspondence to: Dr Martina Delbeck, Cardiology Research, Bayer Schering Pharma AG, Aprather Weg 18, 42096 Wuppertal, Germany

E-mail: martina.delbeck@bayerhealthcare.com

Key words: G-protein-coupled receptor 30-deficient mice, leftventricular contractility, relaxation
$\mathrm{G}$ protein-coupled receptor that has been reported to act as a non-classical membrane-bound estrogen receptor $(3,4)$, although current experimental evidence does not unequivocally support this hypothesis (5). An analysis of LacZ reporter mice demonstrated GPR30 protein expression in the endothelial cells of small arteries in multiple tissues and in the smooth muscle cells and pericytes of brain vessels (6). In line with this expression pattern, recent evidence from two different GPR30-deficient mouse models supports a role for GPR30 in the regulation of vascular tone $(7,8)$. Whereas Haas et al reported that the vasodilatory effects of the GPR30-selective compound G1 were abolished in vessels from GPR30-deficient mice from both genders (7), Martensson et al observed an increased mean arterial blood pressure in female, but not male, GPR30 mutant mice (8). Using a different targeting strategy, we previously generated a third GPR30-deficient mouse model (9). In these mice, we did not observe any involvement of GPR30 in the mediation of estrogenic responses in the classical estradiol target organs, such as the ovary, uterus, mammary gland and hypothalamic-pituitary-gonadal axis (9). Prompted by the findings describing vascular expression of GPR30 (6), we decided to further analyse cardiovascular function in our GPR30-deficient mouse strain (9). Here, we demonstrate that male, but not female, GPR30-deficient mice suffer from impaired left-ventricular cardiac function.

\section{Materials and methods}

GPR30-deficient mice. GPR30-deficient mice used in this study had a C57BL/6 genetic background and lacked the complete open reading frame of GPR30 (9). Experimental animals were derived from heterozygous intercrosses. Mice were individually housed under climate-controlled conditions with a 12-h light/12-h dark cycle and ad libitum access to standard food and water.

Experiments were performed in accordance with the European Community guidelines for experimental animal use and the German law for animal protection.

Histological analysis. Hearts from 5-month-old wild-type and mutant mice ( $\mathrm{n}=4$ per genotype) were fixed overnight in 
A

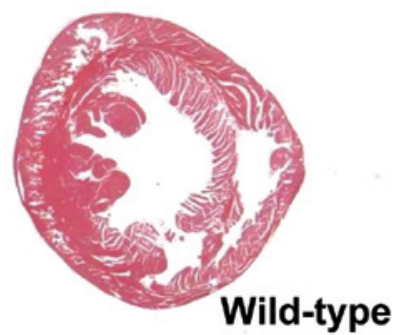

C

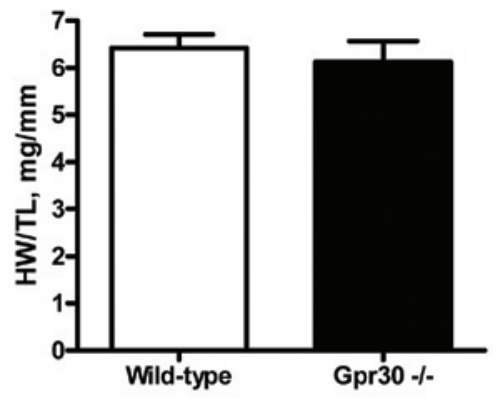

E

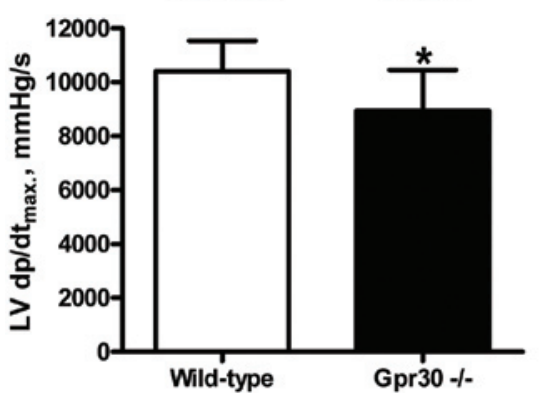

G

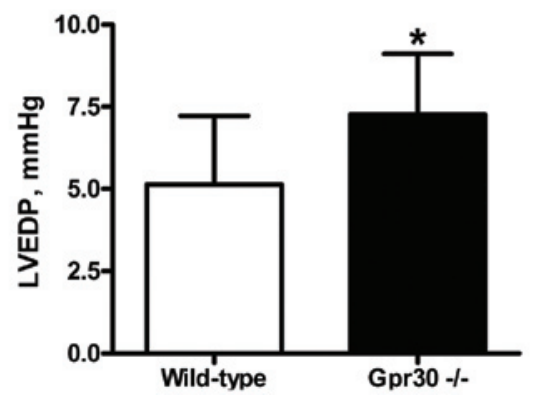

B

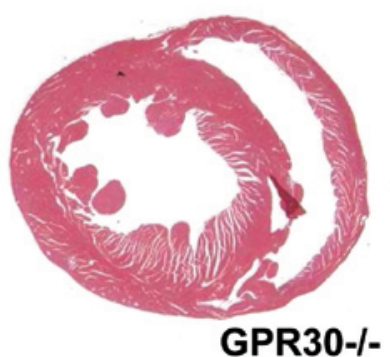

D

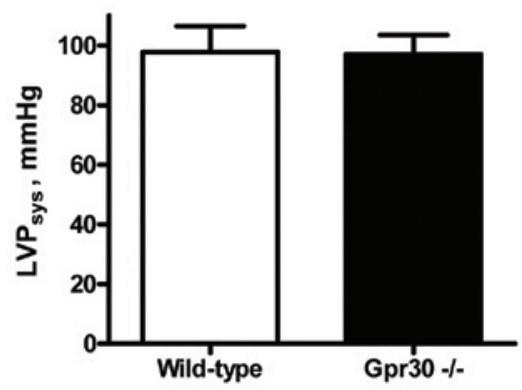

$\mathbf{F}$

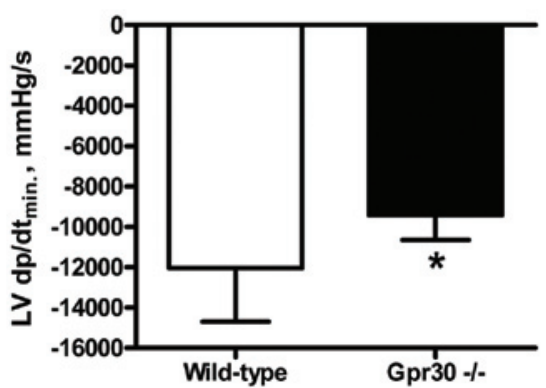

$\mathbf{H}$

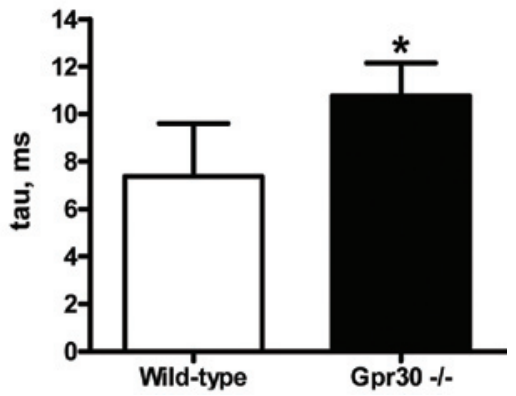

Figure 1. Cardiovascular analysis of male GPR30-deficient mice. (A and B) Transversal heart sections. (C) Heart Weight/Tibia Length (HW/TL). (D) Left ventricular systolic pressure (LVPsys). (E) Maximum rate of left ventricular pressure rise (LVdP/dtmax). (F) Maximum rate of left ventricular pressure decline (LVdP/dtmin). (G) Left ventricular enddiastolic pressure (LVEDP). (H) Relaxation time constant (tau). Data are presented as the mean \pm SD ( $=8-11 /$ group). ${ }^{*} \mathrm{p}<0.05$ vs. wild-type.

$4 \%$ buffered formalin and processed for paraffin embedding. Sections $(7 \mu \mathrm{m})$ were stained with hematoxylin/eosin.

Hemodynamic measurements and tissue sampling. Hemodynamic measurements were performed in 4- to 5-month-old male GPR30-deficient mice $(n=11)$ and their wild-type littermates $(n=8)$. Under isoflurane anesthesia, mice were placed on a thermally controlled heating pad to maintain their body temperature at $37^{\circ} \mathrm{C}$. A Millar microtip catheter (SPR-671, FMI Föhr Medical Instruments GmbH, Seeheim/ Ober-Beerbach, Germany) was inserted through the right carotid artery into the left ventricle for the measurement of left ventricular systolic pressure $\left(\mathrm{LVP}_{\mathrm{sys}}\right)$, left ventricular enddiastolic pressure (LVEDP), relaxation constant (tau), maximum rate of left ventricular pressure rise $\left(\mathrm{LVdP} / \mathrm{dt}_{\max }\right)$, and maximum rate of left ventricular pressure decline (LVdP/ $\left.\mathrm{dt}_{\text {min }}\right)$. Heart rate was measured using echocardiography (ECG). Hemodynamic measurements were analysed with a Power Lab System using Chart 5.0 Software (AD Instruments $\mathrm{GmbH}$, Spechbach, Germany). After exsanguination, the heart and lungs were excised, atria were trimmed away, and the ventricles and lungs were weighed.

Voluntary wheel-running activity. GPR30-deficient mice and their wild-type littermates (4-5 months of age, $n=5$ per group) were individually housed in cages equipped with a running wheel. After a one-week training period, the running distance and top speed were monitored daily during the active night phase from 6:00 p.m. to 8:00 a.m. over a period of three weeks. Microsoft Excel was used for data processing. 
Statistical analysis. Data were displayed graphically using means and standard deviations. Differences in the heart rate, heart weight and lung weight normalized to tibia length between genotypes were assessed using two-sided t-tests. Other hemodynamic parameters were assessed using one-sided t-tests. To analyse the voluntary running wheel behavior, adjusted areas under the response curve (adjusted AUCs) were calculated from day 0 to 21 for all animals. To compare the two genotypes with respect to the adjusted AUCs, one-sided t-tests were used. To assess the magnitude of the differences, the appropriate $95 \%$ confidence intervals were calculated. A significance level of 0.05 was used for all analyses. P-values and confidence intervals were not adjusted for multiplicity; therefore these are only interpreted as exploratory measures. All analyses were performed using $\mathrm{SAS}^{\circledR}$ version 9.1.3.

\section{Results}

Histopathological analysis of organs from 5-month-old GPR30-deficient mice revealed that the left ventricles from GPR30 male (Fig. 1B), but not female, mutant mice appeared enlarged compared to those of their wild-type littermates (Fig. 1A). There was no evidence of malformations of the valves or the cardiac outflow tracts. Hearts from male mutant mice did not exhibit enhanced fibrosis or apoptosis compared to the hearts from the wild-type mice (data not shown).

Heart weights normalized to tibia length (Fig. 1C) and left ventricular systolic pressure (Fig. 1D) did not differ significantly between GPR30-deficient mice and their wild-type littermates. Nevertheless, the hemodynamic characterization of left ventricular function revealed a significant reduction in contractility, denoted by a $14 \%$ decrease in the maximum rate of left ventricular pressure rise in GPR30 mutant mice $(\mathrm{p}=0.0165)($ Fig. 1E). The relaxation capacity (Fig. 1F and H) of the mutant mice was also decreased. In comparison to their wild-type littermates, mutant males exhibited a significant decrease of the maximum rate of left ventricular pressure decline of $2624 \mathrm{mmHg} / \mathrm{s}$ ( $\mathrm{p}=0.0051$ ) (Fig. 1F), with a lower limit of the difference (one-sided $95 \%$ confidence interval) of $\sim 1047 \mathrm{~mm} \mathrm{Hg} / \mathrm{s}$. The relaxation constant tau was significantly increased in mutant hearts by $3.4 \mathrm{~ms}$, with a lower limit of $2 \mathrm{~ms}$ ( $\mathrm{p}=0.0004)$ (Fig. 1H). This led to increased left ventricular end-diastolic pressure (Fig. $1 \mathrm{G}$ ), which was estimated to be $2.13 \mathrm{~mm} \mathrm{Hg}$ higher in mutant animals than in their wildtype littermates, with a lower limit of $0.55(\mathrm{p}=0.0155)$.

By contrast, hearts from female mutant mice did not reveal any functional impairment (data not shown). Lung weights normalized to tibia lengths did not differ significantly between genotypes (wild-type, $8.0 \pm 0.2 \mathrm{mg} / \mathrm{mm}$ vs. GPR30-deficient, $7.9 \pm 0.1 \mathrm{mg} / \mathrm{mm} ; \mathrm{p}=0.51)$ indicating the absence of lung edema. The heart rate of male mutant mice $(463 \pm 66 \mathrm{bpm})$ was slightly, but $n$ ot conclusively, lower than that of their wild-type littermates $(507 \pm 45, \mathrm{p}=0.12)$. Results from the running wheel analysis are depicted in Fig. 2. Daily running distance and top speed were significantly reduced in male (Fig. 2A and B) and female (data not shown) GPR30-deficient mice compared to their wild-type littermates. To rule out the possibility that the observed left-ventricular dysfunction was the result of systemic or metabolic disease, the following parameters were analysed in 6-month-old male mice: oral
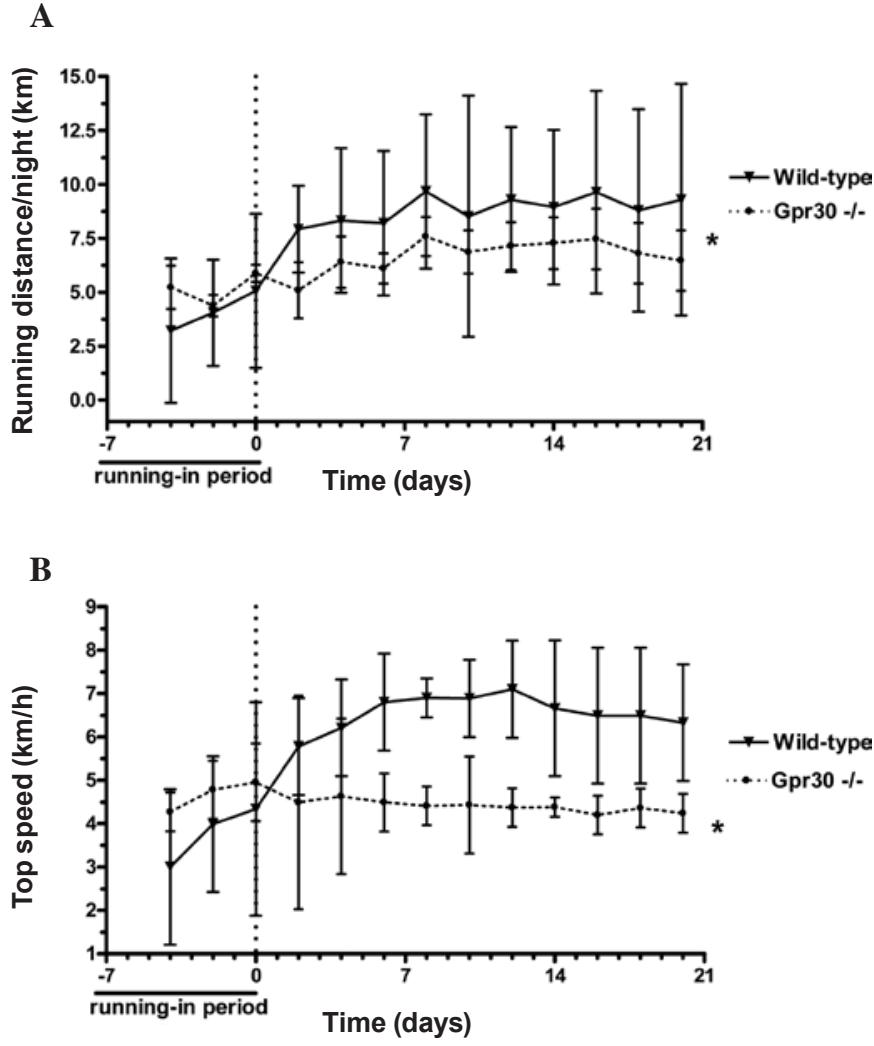

Figure 2. Voluntary wheel-running activity in male wild-type and GPR30-deficient mice. (A) Daily running distance. (B) Top speed. Data are presented as the mean $\pm \mathrm{SD}$ ( $\mathrm{n}=5$ /group). ${ }^{*} \mathrm{p}<0.05$ vs. wild-type.

glucose tolerance test, urine analysis (leukocytes, nitrite, urobilinogen, protein, $\mathrm{pH}$, ketone bodies, bilirubin, glucose), blood parameters (triglycerides, cholesterin, bilirubine, creatinin, phosphorus, calcium, white and red blood cell parameters). There was no significant difference in any of these readout paradigms between the two genotypes (data not shown).

\section{Discussion}

In this study, we identified GPR30 as a novel molecular target that is potentially involved in the gender-specific aspects of cardiovascular disease. Male, but not female, GPR30-deficient mice suffered from mild left-ventricular cardiac dysfunction. Both left-ventricular contractility and relaxation capacity were impaired. In addition, we observed that mutant mice of both genders displayed reduced running wheel activity. However, it would be premature to interpret this phenotype as evidence of an impaired exercise capacity in male mice due to left-ventricular failure, since mutant female mice did not suffer from impaired cardiac function and yet exhibited reduced running wheel activity. Most likely, inactivation of GPR30 within the central nervous system has a direct effect on locomotion in the mutant male and female mice.

For three main reasons, we speculate that the cardiac phenotype observed in male GPR30-deficient mice was not a direct consequence of the cardiac inactivation of GPR30. First, the phenotype was rather mild. Second, previous studies using a GPR30-LacZ reporter mouse described GPR30 expression 
in the vascular beads of small arteries, including the vasa vasorum of the aorta, but not in cardiac myocytes (6). Third, GPR30 mRNA was reported to be up-regulated in endothelial cells in response to shear stress (10).

Presently, four GPR30-deficient mouse models are available, which were generated using different targeting strategies. The phenotypes of these mouse models only partially overlap. Different targeting strategies, breeding schemes, genetic backgrounds and the presence or absence of a neomycin selection marker in the murine genome may have resulted in additional phenotypes, which are not always GPR30-related (5). Nevertheless, experimental evidence from two of the mouse models indicates that GPR30 influences vascular tone $(7,8)$. An increased media-to-lumen ratio in small resistance arteries and increased MAP were described in 9-month-old female GPR30 mutant mice (8). In a second mouse model, ex vivo relaxation of preconstricted carotid arteries in response to the GPR30-selective ligand G1 was abolished in the mutant vessels of both genders (7). Measurement of systemic blood pressure was not performed in this second mouse model (7). It remains to be determined whether increased vascular resistance caused the slight impairment of left-ventricular cardiac function observed in our mice. However, left ventricular systolic pressure was unchanged in the mutant males, which does not support this hypothesis.

In future experiments, tissue-specific ablation of GPR30 is required to further our understanding of the pathophysiological mechanisms leading to functional left-ventricular impairment in male mice. In conclusion, our results suggest that GPR30 is a novel player in the gender-specific aspects of heart failure.

\section{References}

1. Regitz-Zagrosek V, Lehmkuhl E, Lehmkuhl HB and Hetzer R: Gender aspects in heart failure. Pathophysiology and medical therapy. Arch Mal Coeur Vaiss 97: 899-908, 2004.

2. Ghali JK and Lindenfeld JA: Sex differences in response to chronic heart failure therapies. Expert Rev Cardiovasc Ther 6: $555-565,2008$.

3. Revankar CM, Cimino DF, Sklar LA, Arterburn JB and Prossnitz ER: A transmembrane intracellular estrogen receptor mediates rapid cell signalling. Science 307: 1625-1630, 2005.

4. Filardo E, Quinn Y, Graeber C, Shaw S, Dong J and Thomas P: Activation of the novel estrogen receptor $G$ protein-coupled receptor 30 (GPR30) at the plasma membrane. Endocrinology 148: 3236-3245, 2007.

5. Langer G, Bader B, Meoli L, Isensee J, Delbeck M, Ruiz-Noppinger P and Otto C: A critical review of fundamental controversies in the field of GPR30 research. Steroids 75 603-610, 2010.

6. Isensee J, Meoli L, Zazzu V, Nabzdyk C, Witt H, Soewarto D, Effertz K, Fuchs H, Gailus-Durner V, Busch D, Adler T, Hrabe de Ngelis M, Irgang M, Otto C and Ruiz P: Expression pattern of Gpr30 in LacZ reporter mice. Endocrinology 150: 1722-1730, 2009

7. Haas E, Bhattacharya I, Brailoiu E, et al: Regulatory role of G-protein coupled estrogen receptor for vascular function and obesity. Circ Res 104: 288-291, 2009.

8. Martensson UEA, Salehi SA, Windahl S, et al: Deletion of the G protein-coupled receptor 30 impairs glucose tolerance, reduces bone growth, increases blood pressure, and eliminates estradiolstimulated insulin release in female mice. Endocrinology 150: 687-698, 2009.

9. Otto C, Fuchs I, Kauselmann G, Kern H, Zevnik B, Andreasen P, Schwarz G, Altmann H, Klewer M, Schoor M, Vonk R and Fritzemeier KH: GPR30 does not mediate estrogenic responses in reproductive organs in mice. Biol Reprod 80: 34-41, 2009.

10. Takada Y, Kato C, Kondo S, Korenaga R and Ando J: Cloning of cDNAs encoding $\mathrm{G}$ protein-coupled receptor expressed in human endothelial cells exposed to fluid shear stress. Biochem Biophys Res Commun 240: 737-741, 1997. 